\title{
Proposed Practical Communication Architecture for Automatic Fault Detection and Clearance in Secondary Distribution Power Network
}

\author{
Godfrey Chugulu*, Fatuma Simba ${ }^{* *}$, Suzan Lujara ${ }^{* * *}$ \\ * Department of Electronics and Telecommunications Engineering, College of Information and Communication Technologies, \\ Researcher at the department, P.O.Box 33335, Dar es Salaam, Tanzania \\ ** Department of Computer Science and Engineering, College of Information and Communication Technologies, Researcher \\ and Lecturer at the department, P.O.Box 33335, Dar es Salaam, Tanzania \\ *** Department of Computer Science and Engineering, College of Information and Communication Technologies, Researcher \\ and Lecturer at the department, P.O.Box 33335, Dar es Salaam, Tanzania \\ (gchugulu@gmail.com, fatmasimba@gmail.com, suzylui@gmail.com ) \\ ^Corresponding Author; Godfrey Chugulu, P.O.Box 33335, Dar es Salaam, Tanzania,
}

Tel: +255754 710 649, gchugulu@gmail.com

Received: 10.11.2020 Accepted:17.12.2020

\begin{abstract}
A versatile yet secure communication architecture is important for smart grid (SG) applications. Most developing countries are currently deploying communication infrastructure for SG implementation. Survey in literature shows automated protection and control is already done in generation, transmission and primary distribution parts of the grid so as to guarantee reliable power supply to customers. However, secondary distribution power network (SDPN) is usually not monitored. Most of the published studies dealing with communication architectures in SDPN have focused on providing solutions for home automations and smart metering applications but little attention is paid to actual power utilities' needs and implementation challenges facing power utilities specifically when detecting and clearing faults automatically in SDPN. Most power utility companies in developing countries and Tanzania in particular face a lot of challenges when dealing with faults management in the SDPN. This is due to poorly planned and unmonitored low voltage infrastructure. In this paper, practical requirements for SDPN communication architectures are established through challenge driven education (CDE) approach and thereafter, a practical communication architecture appropriate for Tanzania Electric Supply Company Limited (TANESCO) to enable automatic and real time faults detection and clearance in SDPN is proposed. Simulation results show that the developed architecture can successfully be used to practically detect and clear faults at SDPN since it introduces an overall end to end average delay of $1.362 \mathrm{~ms}$ which is lower than what is specified by SG application standard delay time for distribution automation $(100 \mathrm{~ms})$.
\end{abstract}

Keywords Communication architecture, smart grid, challenge driven education, secondary distribution power network.

\section{Introduction}

Countries worldwide vastly depend on reliable supply of electricity for significant progress socially and economically. The economic growth of any developing country like Tanzania depends heavily on reliability of electric power supply. Most developing countries still operate on traditional or legacy grid where there is one way power flow from generation points all the way to consumption points as a result consumed energy and generated one are not coordinated hence it takes long time to respond to quick changes in load [1]. This current setup in developing countries poses a real snag to power utility companies' efforts on supplying reliable power to consumers [2][3].

A review on literature shows that distribution substations in both medium and high voltage power networks are 
monitored and controlled by Supervisory Control and Data Acquisition (SCADA) system. On the other hand, the secondary distribution power networks (SDPNs) are mostly not covered by Remote Terminal Units (RTUs) nor field sensors hence unmonitored [4]. Although several efforts to automate secondary part of power distribution networks have been done worldwide, but still most SDPN are not fully automated due to complexity introduced by its configuration [5][6]. To date, power utility companies are used to handling limited number of devices (e.g., hundreds of substations) for monitoring and control. New communications architectures should be introduced into the grid setup so as to handle a myriad of end nodes (eg. sensors, actuators and Intelligent Electronic Devices (IEDs)) which generate huge data needed for automation of distribution feeders and substations. At present, there is no defined access communication architecture which can handle these endpoints which are widely deployed [7]. Implementation of wideband faults location schemes described in [8] depend on reliable communication architecture which is missing at present.

Currently in Tanzania, defects and faults in the SDPN are reported to power utility company mainly by end consumers or sometimes by utility company employees when doing site visits and in rare cases for some selected areas through smart meters installed which automatically send reports when faults like power cut are detected. This setup bring about a lot of inconveniences and unforeseen losses to power consumers/end customers as well as utility company because the time taken since the occurrence of fault to the time a fault is cleared takes long [2], [9].

To make faults detection and clearance process automatic, it is required first to install in SDPN sensors and IEDs (smart actuators, breakers and re-closers). The automation depends highly on a two-way communication architecture which is dimensioned well so as to carry efficiently and successfully data generated by end devices (sensors, smart meters and actuators) to control center and reverse. All these, require a presence of architecture which is aware of huge amount of data generated by these end devices and implement mechanisms intrinsically to handle computing units processors overutilization and traffic congestion in network. Currently, such architecture does not exist [10] [11].

The existing literature still lacks a research work that has studied challenges facing power utilities on how to automate the whole process of faults detection and clearance in SDPN. Most researches have proposed solutions that use technologies that can not be physically realized by power utilities[12]-[14] or have proposed partial solutions without getting actual requirements from utility personnels [15]. Furthermore, most researches have not evaluated their solutions to check if they can timely be used for faults detection and clearance. It is the focus of this paper, to propose a communication architecture that can be implemented by power utilities while addressing all mentioned issues lacking from existing literature.

In this paper, hybrid, versatile communication architecture for facilitating process of detecting and clearing of faults in SDPN has been proposed. This architecture makes use of wired and wireless communication technologies (hence the term hybrid) and supports distributed processing so as to combat high traffic load resulting from myriad of sensors and IEDs installed at the SDPN. Handling high traffic data from field devices had been a challenge which hindered implementation of visibility and control at SDPN. This designed architecture makes use of techniques for traffic reduction and allows distributed processing so as to be physically and practically realizable by power utility.

The remaining part of this paper is structured as follows: Section II discusses the reviews on SG communication architectures, SG communication requirements and corresponding technologies and finally provides reviews on some related researches/works. Section III details the approaches used in requirements gathering and establishment. It also discusses how collected data was analysed. Section IV discusses the design of proposed hybrid communication architecture Section $\mathrm{V}$ details the architecture implementation and provides discussions on results. Finally, section VI provides conclusion for this work and direction of future research work.

\section{SG Communication}

In this section, popular SG communication network architectures, common communication technologies, communication requirements and application requirements are discussed then research works which mostly relate to this work are reviewed.

\subsection{SG Communication Architectures}

SG communication architecture has been divided by most researchers into three different hierarchies. These are Home Area Network (HAN), Neighborhood Area Network (NAN) and Wide Area Networks (WAN). The HAN connects the in-home smart appliances to a common network. NAN can simply be viewed as a collection of a number of HANs. NAN facilitates collection of customers' data in same neighborhood and transmits them to a power utility. Sometimes NAN is referred as Field Area Network (FAN) when field devices such as IEDs are also connected. WAN simply is the one responsible for backhauling data communication. All communication with distribution substation, transmission lines and power plant is carried out through WAN. More details of SG architectures are found in [16], [17]. This work mainly focuses on NAN but includes IEDs installed along the lines to the distribution transformer.

\subsection{SG Communication Technologies}

So many researches have already been conducted by scholars and by developers from industry and they have all concluded that a practical, optimal architecture for SG application has to make use of both wireless and wired communication [18]. Some examples of common wired communication technologies are fiber optics communication, power line communication (PLC), digital subscriber line (DSL) and coaxial cables communication while wireless communication include ZigBee, Wireless Local Area Network (WLAN)/Wireless Fidelity (WiFi), Cellular networks, Z-wave, worldwide interoperability for Microwave Access (WiMAX) and satellite communication [19]. Details 
of these technologies, which SG applications they support and how they are deployed are summarized in Table 1.

Table 1. Communication Technologies for smart grid (source: [20][21])

\begin{tabular}{|l|l|l|l|l|}
\hline Technology & Location & Data Rate & Coverage & SG Applications \\
\hline $\begin{array}{l}\text { Wireless } \\
\text { LAN }\end{array}$ & Local & $1-54 \mathrm{Mbps}$ & $100 \mathrm{~m}-300 \mathrm{~m}$ & $\begin{array}{l}\text { Distribution Automation } \\
\text { (DA) and protection }\end{array}$ \\
\hline Zigbee & Local & $\begin{array}{l}20- \\
250 \mathrm{Kbps}\end{array}$ & $10 \mathrm{~m}-100 \mathrm{~m}$ & HAN devices automations \\
\hline WIMAX & Backhaul & $70 \mathrm{Mbps}$ & Up to $48 \mathrm{~km}$ & $\begin{array}{l}\text { Advanced Metering } \\
\text { Infrastructure }(\mathrm{AMI}) \& \\
\text { DA }\end{array}$ \\
\hline Cellular & Backhaul & $\begin{array}{l}768 \mathrm{~KB} / \mathrm{s}- \\
100 \mathrm{MB} / \mathrm{s}\end{array}$ & Up to $50 \mathrm{Km}$ & $\begin{array}{l}\text { SCADA \& automations in } \\
\text { remote/rural distribution }\end{array}$ \\
\hline Optical Fibre & Backhaul & $\begin{array}{l}1.25 \mathrm{Gbaud}- \\
2.5 \mathrm{Gbaud}\end{array}$ & $\begin{array}{l}\text { Depends on } \\
\text { availability of } \\
\text { Infrastructure }\end{array}$ & $\begin{array}{l}\text { Transmission lines, } \\
\text { Substations }\end{array}$ \\
\hline PLC & $\begin{array}{l}\text { Local \& } \\
\text { Backhaul }\end{array}$ & $\begin{array}{l}10- \\
500 \mathrm{Kbps}\end{array}$ & SDPN & $\begin{array}{l}\text { HAN automations, } \\
\text { AMI/AMR }\end{array}$ \\
\hline
\end{tabular}

There are a number of factors to consider when selecting which communication technology to deploy for a give smart grid application. These factors are latency, bandwidth, cost of implementation and operation, how accessible it is, who owns it and last but not least geographical location/distance [22], [23]. The successful implementation of different Smart Grid applications depends on the choice of communication technology.

\subsection{SG Communication Architecture Requirements}

The successful implementation of SG largely needs constant communication between major parts of the grid namely distribution (primary and secondary), transmission and generation which in turn demands monitoring and control among these parts to meet SG application requirements. The main $\mathrm{SG}$ communication architectures requirements are discussed in many studies [16], [18], [24][29]; the key communication requirements can be summarized into security, bandwidth, quality of service (QoS), reliability, pervasive availability, security, interoperability, low latency and scalability.

The QoS should be guaranteed by SG communication architecture in order to avoid unnecessary blackouts and power disruptions. QoS is usually measured through latency and bandwidth. The deployed communication technologies should be smart enough to differentiate data according to criticality and give higher priority to delay sensitive data during network congestion time. The communication architecture is supposed to operate reliably even during unexpected system failures.

The SG communication architecture should be designed in a way that it resists attacks so as to maintain privacy of data and security. In addition, SG communication architecture should be designed in such a way that it allows flexibility in implementation so that when upgrades are required, they are implemented with very little modification while end users in all the time remain connected.
The SG communication architecture should be designed in a way that it is affordable to end consumers at the same time economically viable to the utility company. This can be achieved through a number of ways like using already existing deployed communication infrastructure. Last but not least, SG architecture designed should make room for interoperability of systems and devices from different vendors [21].

\subsection{Related Works}

Different communication architectures have been researched extensively in literature. Here is the review of selected works most relevant to this paper.

A work by F. Khan et al in [18], T. Sauter and M. Lobashov in [14], and F. Aalamifar et al in [13] have proposed architectures that are centralized and use PLC/GSM as the main communication technology. Centralized architecture is only convenient for network with fewer number of end nodes but SDPN has huge number of nodes. In addition PLC is not practically feasible when encountered with line cuts and outages. GSM also poses challenges as it cannot be owned by utility company.

Pourmirza and Brooke in [12] had built an architecture which separated hardware from the software architecture. Their architecture setup could be adopted to a real power grid network but Bluetooth is practically not viable as access technology for sensors and the design should take considerations of faults detection and clearance in SDPN so as to exhaust complications brought along with two way traffic flow.

Another work by A. Abdrabou in [30] suggested a communication architecture for handling actively the future SDPN. The negative side is it was designed and simulated in a single cell hence the complexity of SDPN was not tested. The architecture also did not specify backbone/core network technologies involved or how and where data were to be stored and processed. 
Work by K. Ahuja and A. Khosla in [7] proposed a way to provide ubiquitous communication in SG. They proposed hierarchical architecture which had fog and cloud computing zones. Fog computing had been introduced to resolve the local anomalies and to offer less complicated computation to end nodes. Their work also provided deep analysis on the best technologies for HAN/NAN and WAN. Their architecture based on transferring smart meters data but in this work the focus was to detect and clear faults in SDPN where smart meters data was also transferred and processed along with data from sensors and actuators. Their work had fog and cloud computing but in this paper, another layer called edge computing is also added. The end devices (smart meters, sensors and actuators) in this work have some computing capability installed.

To the best knowledge of this paper's authors, there is currently no research work in literature that provides a communication architecture specifically designed for faults detection and clearance in SDPN that has at the same time considered practical requirements from a real power utility and addresses complications brought about by myriad of end nodes.

\section{SG Practical Communication Architecture Requirements Analysis}

In this paper, mixed approaches/methods were used. One method involved feasibility studies and requirements gathering through interviews and investigative questions. Another method involved technical designs and analysis. The whole research process incorporated Challenge Driven Education (CDE) approach where every step from problem identification to validating a solution involved all key stakeholders from electrical power grid and academia.

\subsection{Practical Requirements Gathering Through CDE Approach}

CDE is a method that promotes the involvement of different stakeholders from infant stages in developing practical and usable solutions [31], [32]. In this paper, through CDE approach, actual challenges facing energy sector were reviewed and specifically challenges facing Tanzania Electric Supply Company Limited (TANESCO) hindering initiatives to deploy ICT based solutions in SDPN were discussed. Actual challenges and requirements were gathered through meetings with stakeholders, focused group discussions and interviews. In this paper, stakeholders included employees from TANESCO, Ministry of Energy representatives, and finally researchers and scholars from University of Dar es Salaam, College of Information and Communication Technologies (CoICT). All stakeholders were actively involved in problems identifications and fine tuning, requirements establishments and finally validating solutions.

\subsection{Requirements Establishment Process}

In order to establish practical two way communication architecture requirements in SDPN, two main approaches were used. First approach was through document analysis where intensive literature review was done on published studies where communication architectures, communication technologies and specific SG applications were discussed in details. Emphasis was on solutions and applications operating on SDPN. Second approach was through CDE where actual requirements were established through working hand in hand with TANESCO (already detailed in 3.1).

\subsection{Communication Architecture Requirements From Literature}

Firstly, it was established that the communication architecture designed should efficiently transfer huge volumes of sensed data generated by end devices so as to support implementation of SG applications. Secondly, faults related data and messages for control and actuating should be given higher priority in transferring. Thirdly, due to ubiquitous nature of SDN, wireless communication technology is more viable as an access technology to all end nodes but practically hybrid technologies (combination of wired and wireless) are needed for end to end communication architecture implementation. Fourthly, distributed architecture is more feasible due to amount of data collected because centralized architecture can cause congestion/jam as well as avoid risk of having a single point of failure. Last but not least, to avoid network traffic congestion and over utilization, there must be distributed control points/data processing units installed in different layers of architecture for data offloading/processing; in other words, network should be clustered.

\subsection{Communication Architecture Requirements From TANESCO}

It was noted that TANESCO already has monitoring and control systems for managing primary distribution networks and transmission network. TANESCO has also begun to introduce smartness in SDPN through introduction of Geographical Information System (GIS) database to host details of SDPN equipment and customers. TANESCO currently just needs the communication architecture so as to implement automation in SDPN. TANESCO prefers to own the communication architecture due to sensitivity of SG applications supported and data passing through it. Technologies that depend on third party operators like telecom companies are highly discouraged. PLC is also not preferred as a communication technology since the proposed architecture is specifically designed for handling electrical related faults in SDPN including line cuts. Lastly, due to ubiquitous and meshed nature of SDPN, solutions that use wireless technologies seem to be the most viable option for TANESCO.

\subsection{Data Collection}

Varieties of data were collected at TANESCO after conducting field visit and meetings at their office. Some of the data included the raw data files containing GIS details of where there distribution transformers were installed, details of their SDPN wiring, existing infrastructure and location of their distribution control centers. TANESCO proposed that we use one distribution transformer in Dar Es Salaam city called "BBQ village" as a case study/study area. This transformer was chosen because it had electrical lines 
spanning almost evenly in every directions and had many connected customers (143) hence making it favorite for us to test all our scenarios for our study. Figure 1 shows an actual photo of BBQ distribution transformer taken during site survey. This was a preliminary stage where actual data were collected which was incorporated in designing of a proposed architecture. Some of the data collected at site were WiFi signals in the surrounding with their corresponding channels and strengths, location of furthest customer in each direction and location of power for mounting network devices.

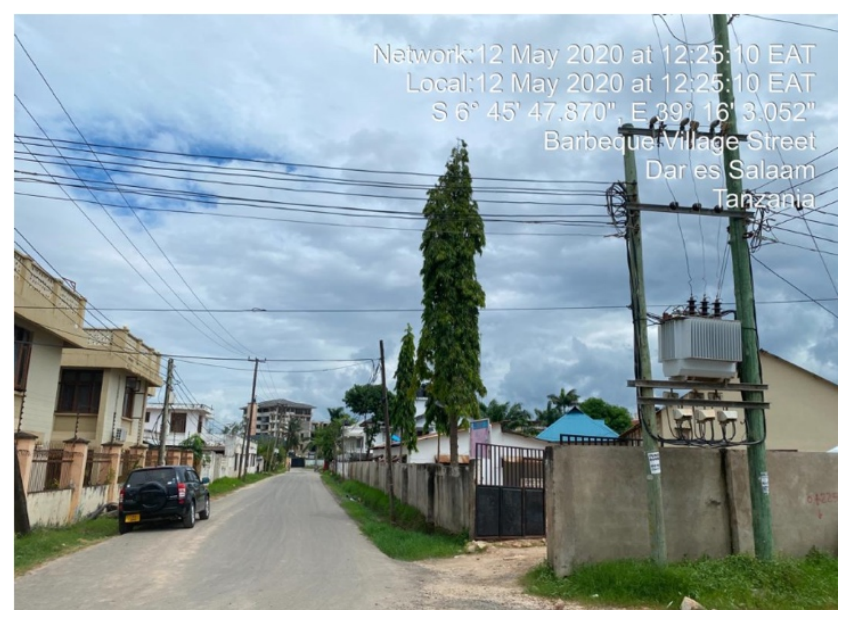

Fig. 1. Study Area SDPN BBQ Village Transformer

Collected shapefile data of TANESCO SDPN at BBQ village was plotted on qGIS software and resulting plot is shown in Figure 2. This plot helps in design stage as it provides an overall picture of actual wiring, branches, sections, lines and location of customers.

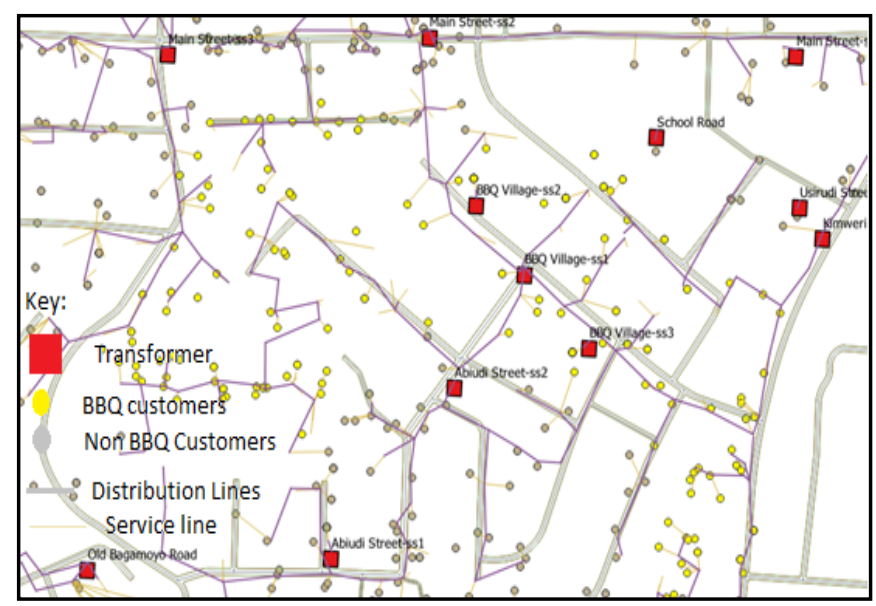

Fig. 2. Actual BBQ Village SDPN Wiring

From stakeholders meetings, it was also found that TANESCO already has an existing fiber ring network which currently is used for backhauling monitoring and control data for transmission and primary distribution network. Same fiber infrastructure can be used for backhauling data from SDPN. For cost effective architecture design, it is required to route SDPN end nodes' data to the nearest TANESCO fiber point of presence (POP). Figure 3 shows main fiber ring connecting substations in Dar es Salaam city and its twelve (12) multiplexers (MUXs) for adding and dropping fiber cables. This data is important since it assists the developer on making design decisions like where to aggregate data etc. For example, the BBQ SS1 transformer in Figure 2 is closest to DCC fiber MUX POP in Figure 3. So all data from BBQ transformer are routed directly to control center which is same location as DCC.



Fig. 3. TANESCO Fiber Ring in Dar es Salaam

\subsection{Data Analysis}

The gathered requirements and data collected for communication architecture design were analyzed through a number of different methods and tools depending on nature or type of the data. Some of the data analysis tools deployed in this work include MS Excel, QGIS, inSSIDER, Mininet$\mathrm{WiFi}$ and application or requirement based parameters comparison. Excel was mainly used in data cleansing and formatting the collected data from utility company. QGIS was used to analyze shapefile raw data collected from TANESCO so as to get meaningful details required for communication architecture design. InSSIDer was used to analyze existing WiFi networks in the study area provided by TANESCO; output after analysis provided key details for access networks design. Mininet-WiFi was used in this phase to analyze/translate text based automated meter reading (AMR) data from TANESCO to an IP packet. Details of size of these packets were required for design phase on the part of dimensioning and bandwidth provision. Application based requirements comparison method was used to analyze those similar requirements captured from published studies against those from bodies of standards. Final derived bandwidth and latency requirements for applications that run to enable distribution automation in SDPN are summarized in Table 2. This latency values in Table 2 are the performance criteria for checking if the developed architecture can actually supports automatic faults detection and clearance.

The communication architecture is evaluated through the application it supports. Popular SG applications in SDPN include Advanced Metering Infrastructure (AMI), Distribution Automations (DA), and Distributed Energy Resources (DER). All these applications have their standard bandwidth and latency requirements. Bandwidth requirement is vital when designing architecture because it allows a researcher to do proper dimensioning. Latency in turn guides 
a designer that the specified time is maximum allowable delay for that specific application to be supported. This is the primary reason why the proposed architecture has been evaluated through its delay/latency imposed.

Table 2. Application Data Requirements

\begin{tabular}{|l|c|c|}
\hline \multicolumn{1}{|c|}{ Application } & Bandwidth & Latency \\
\hline $\begin{array}{c}\text { Advanced Metering } \\
\text { Infrastructure (AMI) }\end{array}$ & $\begin{array}{l}\text { From 10-100 } \\
\text { kbps per node }\end{array}$ & $<2 \mathrm{~s}$ \\
\hline $\begin{array}{c}\text { Distributed Energy } \\
\text { Resource (DER) }\end{array}$ & $56-96 \mathrm{kbps}$ & $<20 \mathrm{~ms}$ \\
\hline $\begin{array}{l}\text { Distribution Grid } \\
\text { Management }\end{array}$ & $\begin{array}{l}\text { Ranges 9.6-100 } \\
\text { kbps depending on } \\
\text { application }\end{array}$ & $<100 \mathrm{~ms}$ \\
\hline
\end{tabular}

\section{Proposed Communication Architecture Design}

\subsection{Architecture Design Overview}

Designing of communication architecture capable of facilitating automatic faults detection and clearance in SDPN based entirely on the requirements already established. The communication architecture was divided into two parts namely the access network and the backbone/core network. Backbone network based on the existing infrastructure (fiber) hence location of fiber POP dictated type of transport technology used. Designing of core network is out of scope of this work since it was assumed that all utility companies already have a well-functioning core network.

Designing access network involved designing coverage area of the network where the geographical area of SDPN was divided into clusters based on location of distribution transformers. Each cluster consist of end nodes (smart meters, smart sensors and smart actuators/switch gears) which are the main data generators in the access network SDPN was modelled based on actual data collected from TANESCO.

Network capacity was dimensioned from data collected and analyzed during requirements establishing and specification stage. Capacity of network links, network switch ports, Access Points (APs) and computing node's port at transformer were dimensioned on full load when all smart meters and sensors were reporting faults on top of routine data sending. For scalability APs, Switches were dimensioned to operate under $75 \%$ capacity utilization. Hence, by aggregation method, cluster and regional capacity could be calculated and provisioned.

\subsection{High Level Design of Communication Architecture}

Architecture was designed based on distributed processing so as to allow both segmentations/clustering and hierarchical processing. Databases are distributed at each computing level (edge, fog and cloud). Edge: end nodes perform crude computing and run basic fault detection algorithms to be able to send data immediately when faults occur or send in predefined routine for historical data. Fog: Cluster computing node works on the data sent from end nodes whether it is fault related or just routine. Each distribution control at the transformer or concentrator has algorithms running in it to handle faults in its area. It receives fault related data and works on them first before publishing them to higher levels (control center). At the end all data, normal or fault related are permanently stored at control center for future use in computations (cloud).

The designed architecture has to handle both faults related and routine data traffic so two traffic patterns were modelled. Routine sensed data were transported via Transmission Control Protocol (TCP) so as to ensure reliable delivery and those sending fault related data were transported via User Datagram Protocol (UDP) so as to meet latency criteria. Regardless of underlying protocols in the applications running in the nodes, all communications in the architecture are IP based; Only IP packets are sent. Protocol change/bundling is not part of architecture developer.

For ease of maintenance, security and reducing communication overhead, static IP addressing was used. Since all end nodes are stationary then these IP addresses can also be used as location identifiers. For security reasons, the communication architecture should not be connected to Internet at all. All the end nodes are hard coded with network access authentication details. Example of other security measures include IEEE 802.11i Wi-Fi Protected Access II (WPA-2) which improves the cyber security through Advanced Encryption Standard (AES) in wireless LANs. Due to sensitivity of data and control, communication architecture designed is fully owned by utility company.

In order to meet challenge of ubiquitous nature of SDPN, latency and bandwidth requirements imposed by applications for facilitating automatic faults detection and clearance, different communication technologies are implemented at different network layers. This makes the architecture hybrid as it involves different technologies, protocols and setups. The architecture is divided in to two layers namely access layer and transport/data backhauling layer. The access layer is part of the network responsible for connecting end nodes (sensors, smart meters and IEDs), access/aggregation switches, access points and computing nodes at cluster level.

On access network, technology used is WLAN/WiFi, all sensors, IEDs and smart meters are equipped with WiFi shield for connecting to the network. Powerful outdoor directional wireless access points installed at the transformer and on selected locations to provide coverage and capacity to end nodes. Main aggregation point in a cluster is at the transformer so all measurement and control data are channeled there. Figure 4 summarizes the high level design of the whole architecture where clustering, distributed computing and hierarchies have been taken into consideration. 
On transport layer, a number of technologies are used to shunt data in the architecture. From aggregation switch to control centre, three different technologies are used namely Ethernet, point to point (P2P) microwave and fiber optic network. TANESCO has fiber ring already implemented which connects all district offices. For cost effective architecture implementation, data from aggregation switch needs to be sent to the nearest fiber POP so that it can reach control centre. When the fiber POP is less than 100 meters from the aggregation switch, outdoor Ethernet cable is used but when the distance from the aggregation switch is more than 100 meters, P2P microwave is used to backhaul data to fiber POP. Fiber optic backhauls data to the control/data centre for processing and storage.

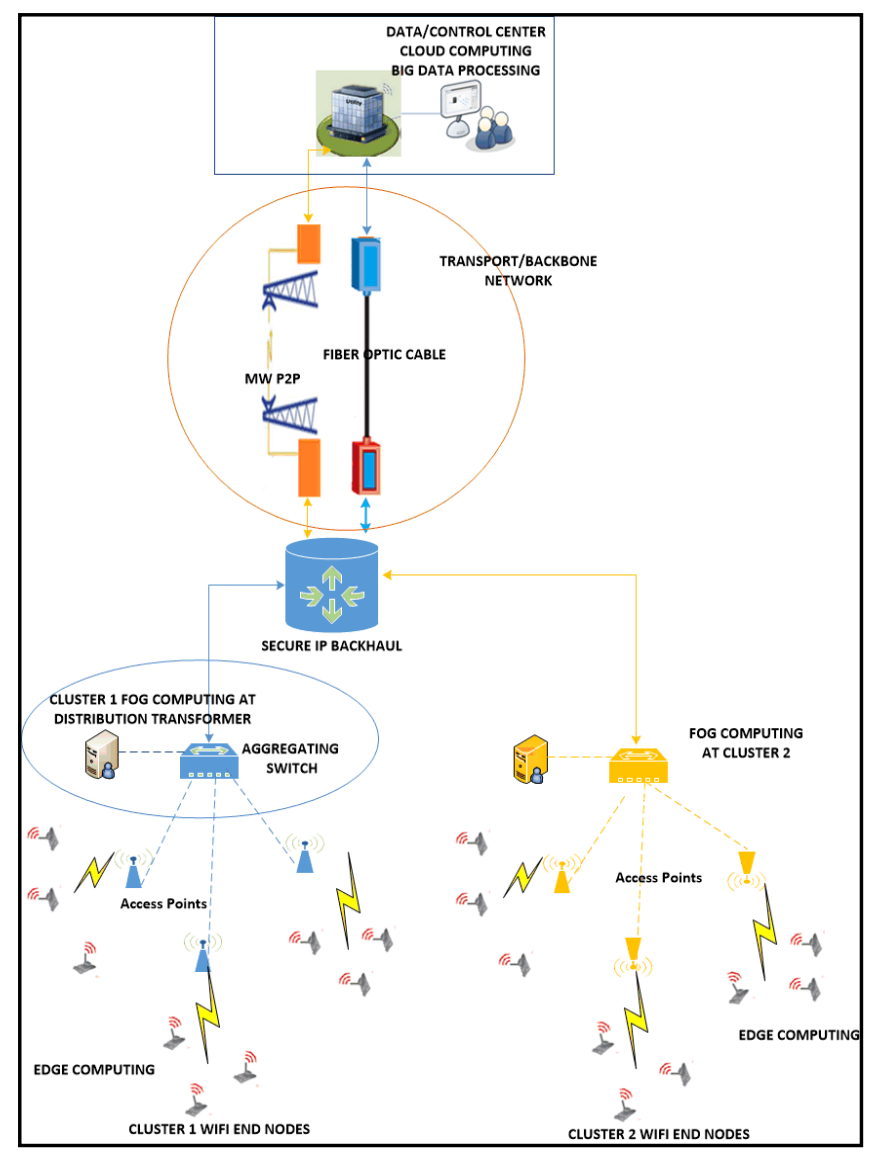

Fig. 4. Practical Overall Communication Architecture Design

\subsection{Study Area Physical Communication Architecture Design}

As already mentioned, physical site survey was conducted to get actual details of the study area and noting any environmental challenges. After technical analysis, here are the design requirements: three directional powerful outdoor access points are needed so as to provide sufficient coverage, an eight ports aggregate switch is needed for interconnecting network devices including a computing device with an Ethernet port and WiFi module, a switch that has an uplink port for backhauling/routing data and at least three spare/idle ports for expansion. APs and switches must be 'open flow' enabled so as to allow running Software Defined Network
(SDN) applications/configurations. Outdoor data and power cabinet $(9 \mathrm{U})$ is also needed for housing these network devices. A $12 \mathrm{~m}$ mounting pole is required for APs/Antennas mounting. Figure 5 shows on site high level design and equipment setup. All equipment are collocated with transformer for security and power availability reasons.

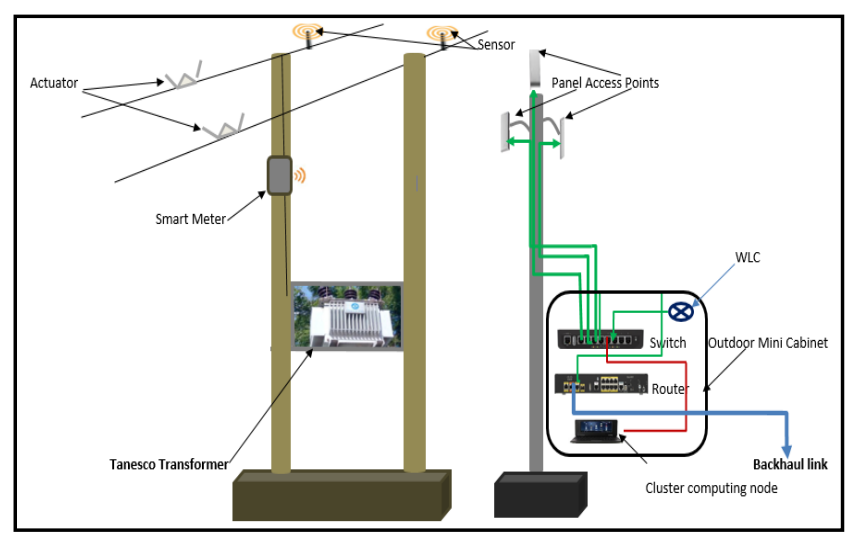

Fig. 5. Study Area Physical Design and Equipment Setup

The top view of APs' mounting and corresponding azimuths is shown in Figure 6. This snapshot was generated from qGIS buccurest software. The design also incorporates a range extender for covering the furthest end node/customer where APs cannot reach. This figure is important as it alerts the designer about on-ground implementation challenges.

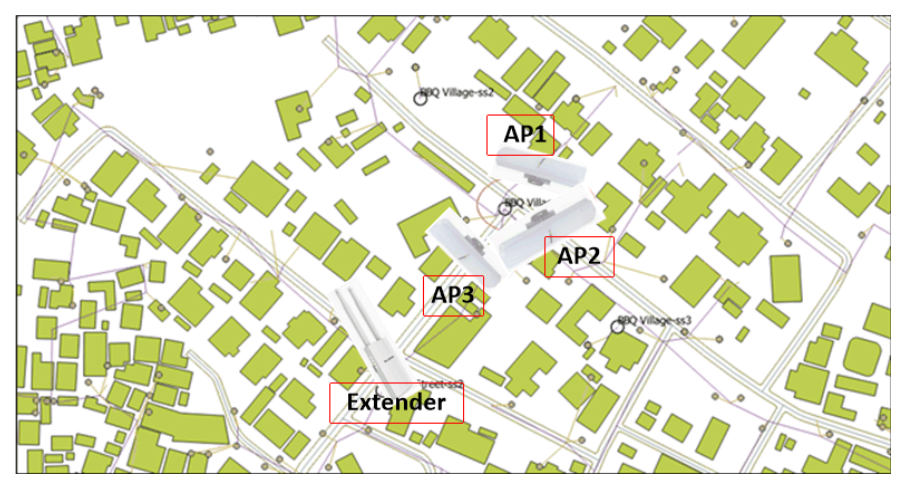

Fig. 6. Actual APs Positioning in Study Area

\section{Implementation and Results Discussion}

\subsection{Architecture Implementation Overview}

The overall communication network architecture was built on packet tracer (PT) emulation tool for visualizing and analysing single packets routing on access network and provide a user friendly cluster based look of network architecture developed. Packet spoofing was implemented on the link to fog machine so as to visualize details of the packets being sent and checking if network details including routing protocols and IP addressing was properly done. Access network was re-modelled on Mininet WiFi simulator so as to be able to simulate real life operations of SDPN, analyse the overall network and implement all scenarios for network validation and evaluation. Architecture was 
modelled based on the data collected and calculated bandwidth on a specified routine as shown in Table 3 . These details were established during requirement analysis stage.

Table 3. Modelling Data

\begin{tabular}{|r|l|l|l|l|l|}
\hline SN & NODE TYPE & APP NAME & BANDWIDTH & DIRECTION & ROUTINE \\
\hline 1 & Smart meter & AMI/AMR & 669 Bytes & UPLINK & Every 20 mins \\
\hline 2 & Sensor & DA/FA & 120 Bytes & UPLINK & $\begin{array}{l}\text { When fault } \\
\text { occurs }\end{array}$ \\
\hline 3 & Actuator & DA/FA & 120 Bytes & UPLINK & on query \\
\hline 4 & Smart meters & DA/FA & 120 Bytes & UPLINK & $\begin{array}{l}\text { When fault } \\
\text { occurs }\end{array}$ \\
\hline 5 & Sensor & DA/FA & 669 Bytes & UPLINK & $\begin{array}{l}\text { Every } 20 \\
\text { seconds }\end{array}$ \\
\hline 6 & $\begin{array}{l}\text { Cluster computing } \\
\text { node }\end{array}$ & DA/FA & 120 Bytes & DOWNLINK & on query \\
\hline & $\begin{array}{l}\text { Cluster } \\
\text { Computing node }\end{array}$ & $\begin{array}{l}\text { Firmware } \\
\text { Update }\end{array}$ & 2024 Bytes & DOWNLINK & $\begin{array}{l}\text { Hrs } \\
\text { Hrs } 02: 00\end{array}$ \\
\hline
\end{tabular}

\subsection{Architecture Modelling on Packet Tracer}

As illustrated in Figure 7, the complete communication architecture was modelled on PT. Three directional APs were installed in each cluster, all these APs were connected to aggregate switch which is connected to a computing node (fog machine) and is also connected to edge router for connecting to a backhauling network. This specific cluster (BBQ Transformer network) had 143 customers which translates to 143 smart meters, 12 optimally deployed smart sensors and 10 smart actuators.
All end nodes in a cluster were assigned static IP address so as to easily identify them uniquely. Strong authentication is applied to all end devices which connect to respective area SSID. All nodes are pre-configured with strong password for hooking to a given SSID; combination of password with MAC address are used for authentication so that to stop unknown devices from connecting/sniffing in the network. Subnet mechanism has been done to segment the large network into blocks of small network so that to avoid large network risk.

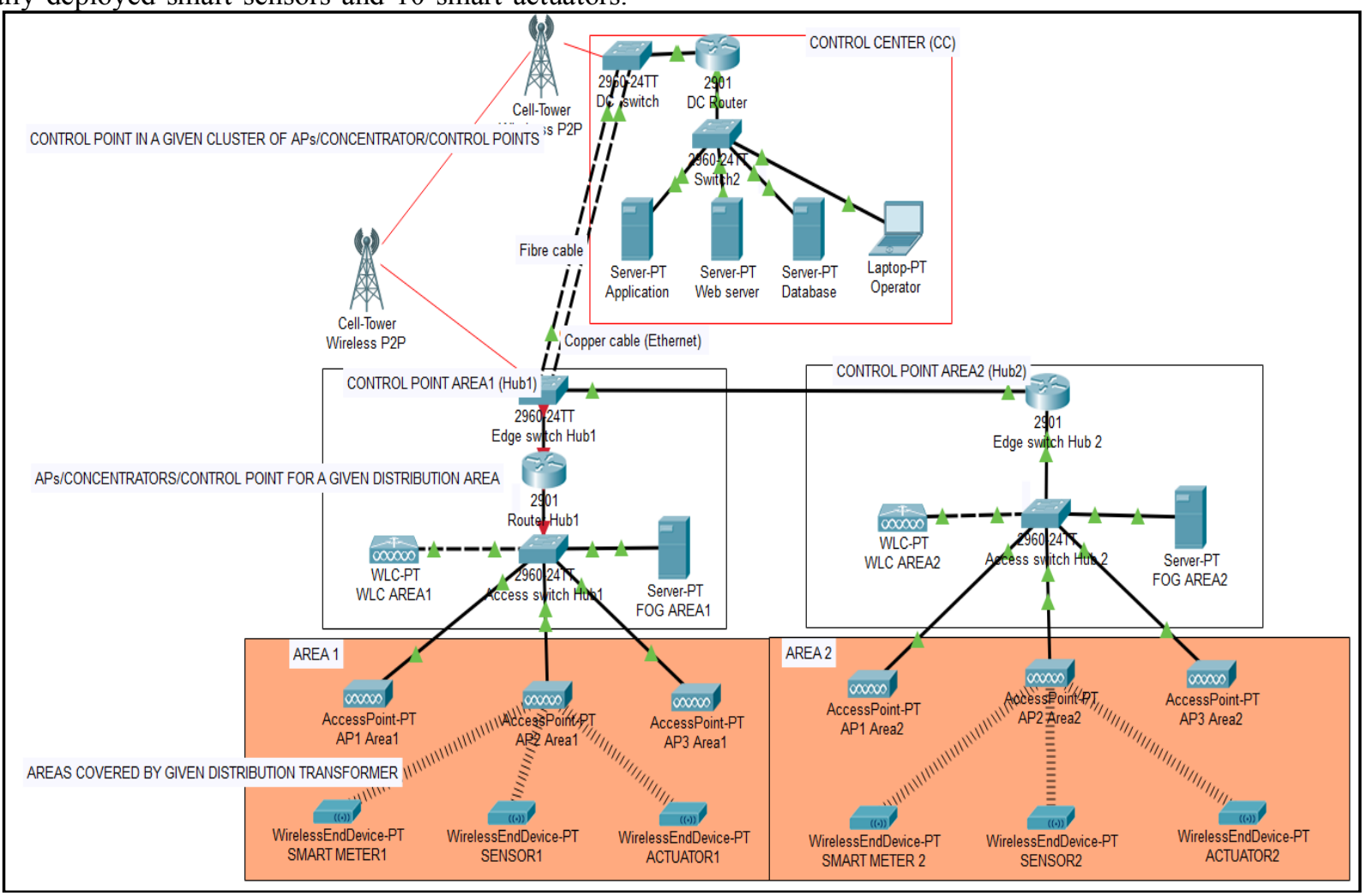

Fig. 7. End to End Communication Architecture Modelled in PT 
After implementation on PT, architecture was tested to check if packets were rightly routed and successfully delivered. Figure 8 shows a screenshot taken from simulation panel of PT when simulation was on progress. It can be seen that different nodes in the network were communicating using implemented protocols successfully.

\begin{tabular}{|c|c|c|c|c|}
\hline \multicolumn{5}{|c|}{ Simulation Panel } \\
\hline \multicolumn{5}{|c|}{ Event List } \\
\hline \multirow[t]{11}{*}{ Vis. } & Time(sec) & Last Device & At Device & Type \\
\hline & 0.008 & AREA2-AP2 & ACTUATOR-7 & ICMP \\
\hline & 0.008 & AREA2-AP2 & SMARTMETE... & ICMP \\
\hline & 0.008 & AREA2-AP2 & SENSOR-28 & ICMP \\
\hline & 0.413 & Access switc... & AREA2-AP1 & HSRP \\
\hline & 0.413 & Access switc... & WLC-AREA2 & HSRP \\
\hline & 0.413 & Access switc... & FOG AREA2 & HSRP \\
\hline & 0.960 & AREA2-AP1 & SENSOR-26 & STP \\
\hline & 0.960 & AREA2-AP1 & SENSOR-27 & STP \\
\hline & 1.272 & WLC-AREA1 & Access Swi... & CAPWAP \\
\hline & 1.273 & Access Switc... & AREA1-AP1 & CAPWAP \\
\hline
\end{tabular}

Fig. 8. Sample Protocols Implemented in PT

\subsection{Architecture Modelling on Mininet - WIFI}

Access network part of the architecture was modelled in Mininet-WiFi simulator. Mininet-WiFi is open source Linux based software defined network (SDN) network emulator specifically for simulating WLAN/WiFi. It has virtualized inbuilt WiFi stations and APs. It has been selected as an emulator for access network in this study because it has recently been extensively researched on and specifically designed for simulating WiFi networks plus it supports open flow protocols in SDN which is the feature of interest in this work so as to allow dynamic configuration and allow running specific solution based applications. For example in [33] it was used for reliability enhancements. Details on Mininet and use cases are here [34].

Network was modelled and implemented in Linux environment through Python programming language. All end nodes were first created by assigning device names, MAC addresses, static IP addresses, and geographical positions. Each task was done through algorithms developed. There were a number of algorithms implemented in this work during this phase; some of them include algorithms for creating nodes, algorithm for assigning IPs and locations, algorithms for creating links and enabling associations and algorithms for modelling a data packet and algorithm for traffic scenarios.

After all end nodes, network nodes, links are created and configured, then the server for wireless medium environment is started and the network is started. When everything is up and running, then interface for 802.11 radio network monitoring (hwsim0) was configured and put up. This interface is the one used to monitor all transmitted frames regardless of channel. Through monitoring and analysing network traffic is how architecture performance is evaluated.

The modelled architecture is versatile as it allows a researcher/user to define custom size of network and analyse. Once every network setup and configurations are done, network simulations are started where a user is prompted to define the size (number of end-nodes and dimensions of the coverage area) of access network. Figure 9 shows the first window that appears when simulation started. A user gets to define his/her custom network.

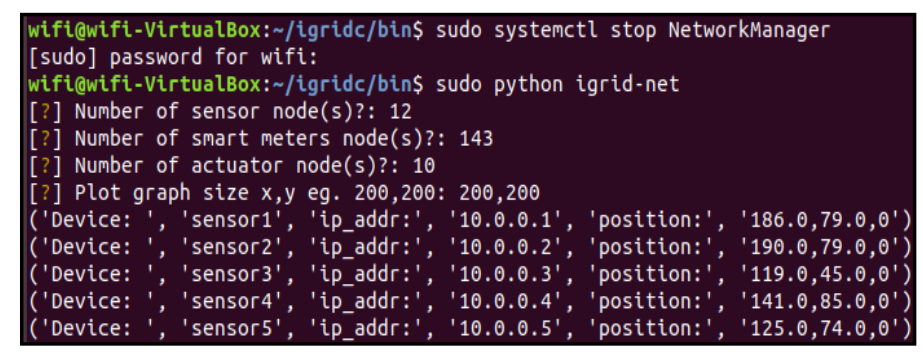

Fig. 9. Custom Access Network Definition Window

In this paper, the cluster provided by TANESCO as a case study had 143 smart meters, 12 smart sensors and 10 smart actuators. These were the details filled in configuration window in Figure 9 plus the coverage plot for testing was defined as a square of size $200 \mathrm{~m} * 200 \mathrm{~m}$ covered by three APs. To show that everything works as configured, Figure 10 shows the Mininet WiFi graph showing how end nodes are located in the coverage area.

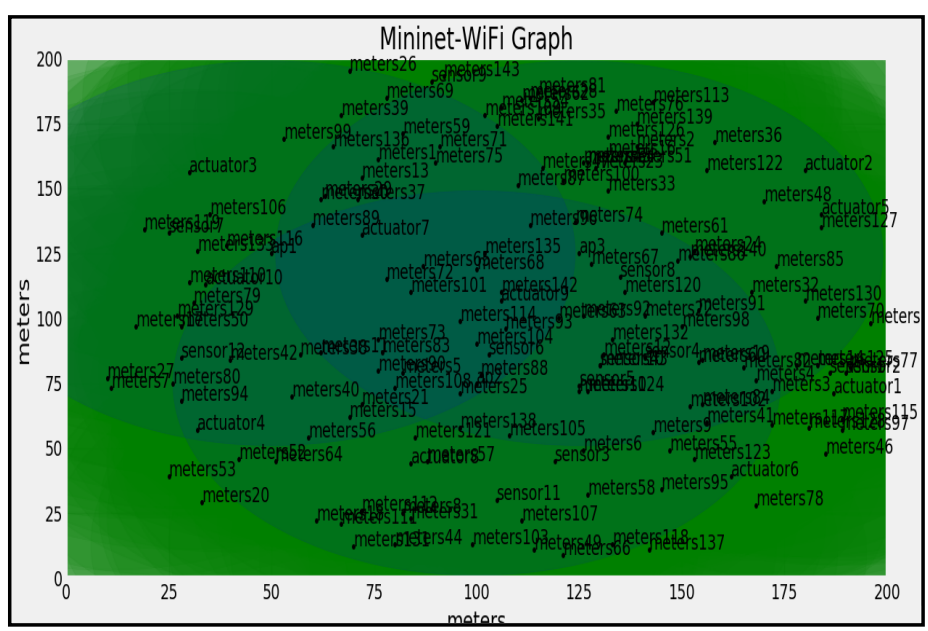

Fig. 10. End Nodes Locations on a Coverage Plot

\subsection{Simulation Results Discussion}

Different scenario were run so as to check if the designed architecture can actually be used to facilitate automatic detection and clearance of faults at SDPN. First scenario was to check if all end nodes in the architecture send data as pre-defined when there is no fault. The results are 
stipulated in Figure 11 which shows actuators (green line), sensors (red line) and smart meter (blue line) sending data in periodic manner. Smart meters and sensors data are higher as they send real sensed data while actuators data is relatively low because they just respond to commands. All these data are sent via TCP protocol. Data is sent in predefined intervals so as not to overwhelm the network bandwidth.

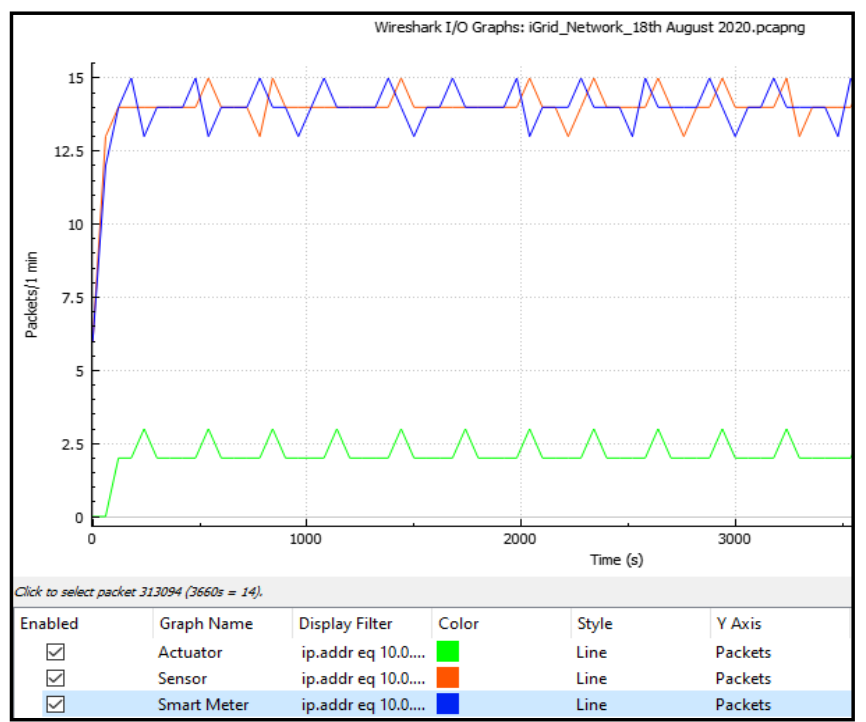

Fig. 11. Actuators, Sensors and Smart Meters Sending Periodic Data

When fault occurs in a specific section in a cluster or in the whole cluster, all the end nodes involved in fault detected area 'need to send data immediately. For these nodes to meet the latency criteria, the fault related data must be sent via UDP protocol as TCP with its acknowledgements would delay. When fault has occurred, some nodes from sections not involved in fault keep on sending TCP routine data while those involved in fault send fault related data via UDP protocol. Figure 12 shows UDP data on top of TCP data sent in the architecture during occurrence of a fault.

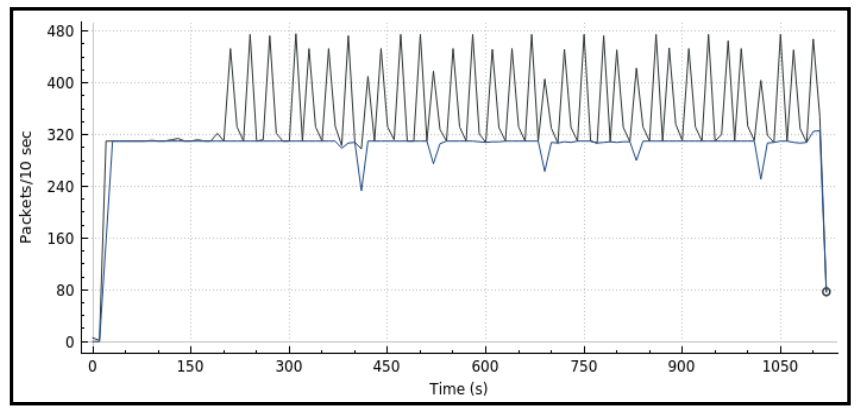

Fig. 12. UDP Data Packets On Top of TCP Data Packets

Now to test if the designed architecture can really detect faults timely, round trip time (RTT) is used for verification. Firstly, while network was running normally, another sporadic data was sent from a smart meter (meters140) to a cluster controlling node $(10.10 .10 .254)$ to check how long it takes for data to be successfully delivered to destination and acknowledged. From Table 3, a smart meter routine data packet has 669 Bytes (containing 20 separate readings); 10 packets of 669 Bytes each were sent to verify RTT and results are summarized in Figure 13. Lowest RTT is $0.662 \mathrm{~ms}$, maximum RTT is $3.68 \mathrm{~ms}$ and the average is $1.561 \mathrm{~ms}$. From Table 2, AMI acceptable latency is $2 \mathrm{~s}$, which means the imposed delay by the designed architecture is within the acceptable value range.

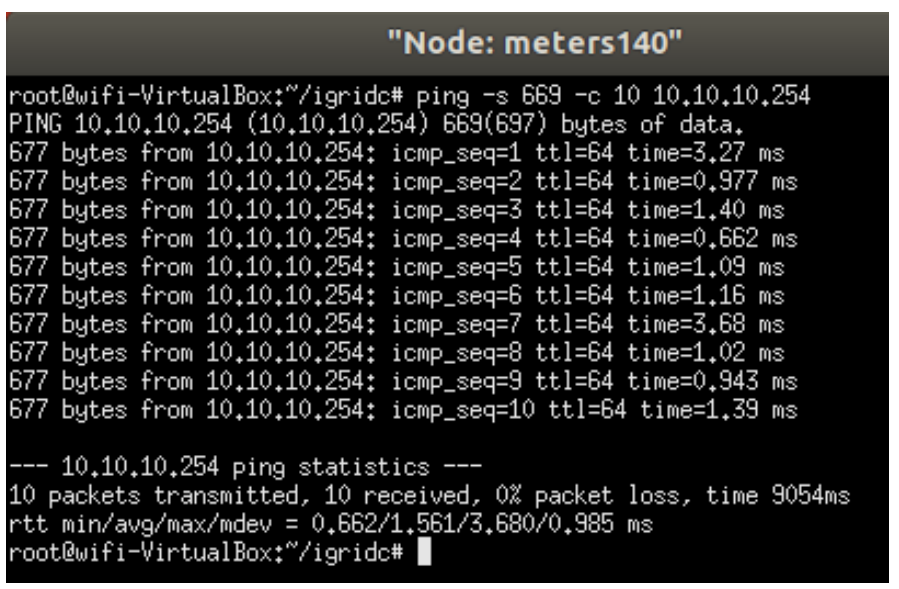

Fig. 13. Smart Meter RTT Details during Normal Operations

To verify how soon can faults be detected, a sensor is used to send testing packets with size exactly like PDUs containing fault information; 10 separate packets were sent and the summary of RTT is provided in Figure 14. Average propagation delay imposed by design is $1.362 \mathrm{~ms}$ which is lower than $20 \mathrm{~ms}$ required for DER automations and $100 \mathrm{~ms}$ for distribution automations specified in Table 2 .

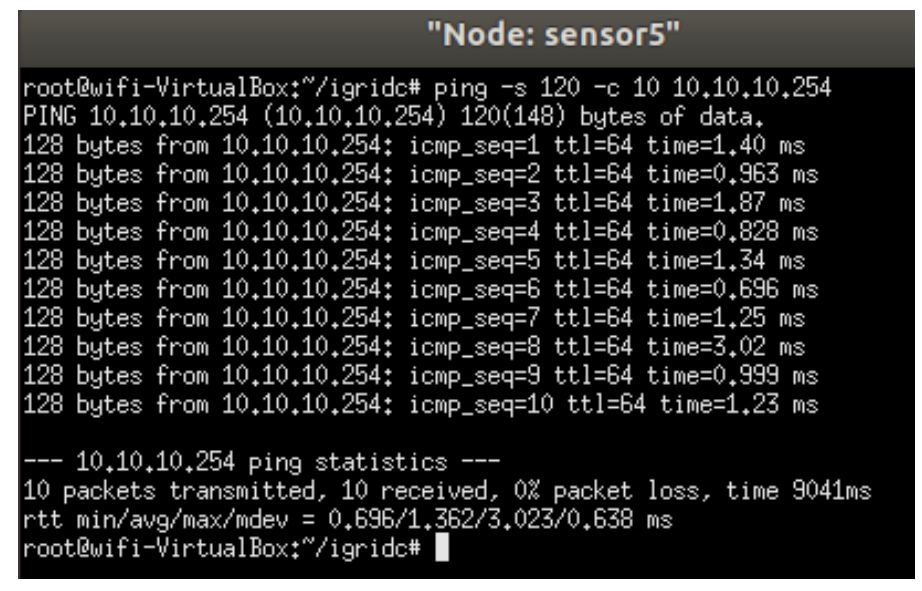

Fig. 14. Sensor RTT Details during Fault

The key feature of the design is to allow distributed processing and move processes/computation as much as possible close to end nodes so as to make bandwidth and latency requirements feasible. In this paper, all cluster processing is handled by fog node at the transformer 
location. All RTT scenarios are tested between end nodes and fog node. These simulation results indicate that if the design were to be realized physically, TANESCO can timely be able to detect and clear faults automatically in SDPN.

\section{Conclusion and Further Research}

In this paper, the communication architecture has been proposed. This architecture is clustered, makes use of distributed processing and uses feasible hybrid communication technologies that can be implemented by real power utility. Unlike previous researches, this work incorporated actual 'user requirements' by power utility company. This study has also contributed understanding of actual communication architecture needs and wishes of a power utility company through $\mathrm{CDE}$ approach. CDE approach is another contribution to the body of knowledge as it provides platform for incorporating actual requirements from industry. It's the methodology that is not usually found in traditional researches.

In future work, we plan to develop the designed architecture on a pilot site (physical prototype) and analyse challenges imposed by real SDPN outdoor environment.

\section{Acknowledgements}

The ongoing iGrid Research Project at the University of Dar es salaam (UDSM) is financed by Swedish International Development Agency (SIDA). This paper is among the outcome of iGrid Research Project and authors are appreciative to SIDA for their financial kindness and thankful to TANESCO for accepting to be part of this iGrid project through CDE approach.

\section{References}

[1] I. Colak, R. Bayindir, and S. Sagiroglu, "The Effects of the Smart Grid System on the National Grids," 8th Int. Conf. Smart Grid, icSmartGrid 2020, pp. 122-126, 2020, doi: 10.1109/icSmartGrid49881.2020.9144891.

[2] D. Mnyanghwalo, S. Kawambwa, R. Mwifunyi, G. M. Gilbert, D. Makota, and N. Mvungi, "Fault Detection and Monitoring in Secondary Electric Distribution Network Based on Distributed Processing," 2018 20th Int. Middle East Power Syst. Conf. MEPCON 2018 - Proc., pp. 84-89, 2019, doi: 10.1109/MEPCON.2018.8635141.

[3] M. Garba, M. A. Tankari, and G. Lefebvre, "Methodology for Analyzing of a Grid Weakness and Resiliency Factors - Case of Niger National Grid," 7th Int. IEEE Conf. Renew. Energy Res. Appl. ICRERA 2018, vol. 5, pp. 1259-1265, 2018, doi: 10.1109/ICRERA.2018.8566870.

[4] A. Abdrabou, "A Wireless Communication Architecture for Smart Grid Distribution
Networks," doi: 10.1109/JSYST.2014.2304291.

[5] A. Zidan et al., "Fault Detection, Isolation, and Service Restoration in Distribution Systems: Stateof-the-Art and Future Trends," IEEE Trans. Smart Grid, pp. 1-16, 2016, doi: 10.1109/TSG.2016.2517620.

[6] Y. Andegelile, N. Mvungi, and M. Kissaka, "SDN Based High Availability Communication Network Architecture for Secondary Distribution Electric Power Grid," 7th Int. Conf. Smart Grid, icSmartGrid 2019, pp. 52-57, 2019, doi: 10.1109/icSmartGrid48354.2019.8990828.

[7] K. Ahuja and A. Khosla, "Network selection criterion for ubiquitous communication provisioning in smart cities for smart energy system," J. Netw. Comput. Appl., vol. 127, pp. 8291, 2019, doi: 10.1016/j.jnca.2018.11.011.

[8] F. M. Aboshady, M. Sumner, and D. W. P. Thomas, "A Wideband Fault Location Scheme for Active Distribution Systems," 7th Int. IEEE Conf. Renew. Energy Res. Appl. ICRERA 2018, vol. 5, pp. 891-896, 2018, doi: 10.1109/ICRERA.2018.8566780.

[9] G. Chugulu and F. Simba, "Communication Architecture for Automatic Faults Detection and Clearance in Secondary Distribution Power Grid: The Case of TANESCO," Proc. 2019 7th Int. Conf. Smart Energy Grid Eng. SEGE 2019, pp. 223-229, 2019, doi: 10.1109/SEGE.2019.8859909.

[10] Y. Andegelile, G. Chugulu, H. Mbembati, A. Bitebo, and H. Kundaeli, "Enhancing Faults Monitoring in Secondary Electrical Distribution Network," in I5th IFIP WG 9.4 International Conference on Social Implications of Computers in Developing Countries, 2019, pp. 1-804, doi: 10.1007/978-3-030-18400-1 58.

[11] D. Baimel, S. Tapuchi, and $\overline{\mathrm{N}}$. Baimel, "Smart grid communication technologies- overview, research challenges and opportunities," 2016 Int. Symp. Power Electron. Electr. Drives, Autom. Motion, pp. 116-120, 2016, doi: 10.1109/SPEEDAM.2016.7526014.

[12] Z. Pourmirza and J. M. Brooke, "A Realistic ICT Network Design and Implementation in the Neighbourhood Area of the Smart Grid," Smart Grid Renew. Energy, vol. 4, no. 6, pp. 436-448, 2013.

[13] F. Aalamifar, H. S. Hassanein, and G. Takahara, "Viability of powerline communication for the smart grid," in 2012 26th Biennial Symposium on Communications, QBSC 2012, 2012, doi: 10.1109/QBSC.2012.6221343.

[14] T. Sauter and M. Lobashov, "End-to-end communication architecture for smart grids," IEEE Trans. Ind. Electron., vol. 58, no. 4, pp. 12181228, 2011, doi: 10.1109/TIE.2010.2070771.

[15] A. Abdrabou, "A Wireless Communication Architecture for Smart Grid Distribution Networks," pp. 1-11, 2014. 
[16] Z. Soufiane, B. Slimane, and E. N. Abdeslam, “A synthesis of communication architectures and services of smart grid systems," Proc. - $20163 \mathrm{rd}$ Int. Conf. Syst. Collab. SysCo 2016, 2017, doi: 10.1109/SYSCO.2016.7831334.

[17] N. Raza, M. Q. Akbar, A. A. Soofi, and S. Akbar, "Study of Smart Grid Communication Network Architectures and Technologies," J. Comput. Commun., vol. 07, no. 03, pp. 19-29, 2019, doi: 10.4236/jcc.2019.73003.

[18] F. Khan, A. ur Rehman, M. Arif, M. Aftab, and B. $\mathrm{K}$. Jadoon, "A survey of communication technologies for smart grid connectivity," 2016 Int. Conf. Comput. Electron. Electr. Eng. (ICE Cube), pp. 256-261, 2016, doi: 10.1109/ICECUBE.2016.7495234.

[19] P. Rengaraju, C.-H. Lung, and A. Srinivasan, "Communication requirements and analysis of distribution networks using WiMAX technology for smart grids," 2012 8th Int. Wirel. Commun. Mob. Comput. Conf., pp. 666-670, 2012, doi: 10.1109/IWCMC.2012.6314284.

[20] Y. Tsado, K. Gamage, and D. Lund, "Communication Technologies for Smart Grid Ubiquitous Sensor Network System," no. May, pp. 13-17, 2013.

[21] F. Ayadi, I. Colak, and R. Bayindir, "Interoperability in Smart Grid," 7th Int. Conf. Smart Grid, icSmartGrid 2019, pp. 165-169, 2019, doi: 10.1109/icSmartGrid48354.2019.8990680.

[22] P. P. Parikh, M. G. Kanabar, and T. S. Sidhu, "Opportunities and challenges of wireless communication technologies for smart grid applications," IEEE PES Gen. Meet., no. Cc, pp. 1-7, 2010, doi: 10.1109/PES.2010.5589988.

[23] A. Patel, J. Aparicio, N. Tas, M. Loiacono, and J. Rosca, "Assessing communications technology options for smart grid applications," 2011 IEEE Int. Conf. Smart Grid Commun., pp. 126-131, 2011, doi: 10.1109/SmartGridComm.2011.6102303.

[24] S. Afsar, G. Nadendla, and R. Sudha, "A Review of Recent Development in Smart," pp. 96-103, 2015.

[25] B. M. Buchholz and Z. A. Styczynski, "Communication requirements and solutions for secure power system operation," in 2007 IEEE Power Engineering Society General Meeting, PES, 2007, doi: 10.1109/PES.2007.385633.

[26] M. Daoud and X. Fernando, "On the Communication Requirements for the Smart Grid," Energy Power Eng., 2011, doi: 10.4236/epe.2011.31008.

[27] J. Gao, Y. Xiao, J. Liu, W. Liang, and C. L. P. Chen, "A survey of communication/networking in Smart Grids," Futur. Gener. Comput. Syst., vol. 28, no. 2, pp. 391-404, 2012, doi: 10.1016/j.future.2011.04.014.

[28] C. Pirak, T. Sangsuwan, and S. Buayairaksa, "Recent Advances in Communication
Technologies for Smart Grid Application: A Review," Proc. Int. Electr. Eng. Congr., pp. 3-6, 2014, doi: 10.1109/iEECON.2014.6925952.

[29] V. K. Sood, D. Fischer, J. M. Eklund, and T. Brown, "Developing a communication infrastructure for the smart grid," in 2009 IEEE Electrical Power and Energy Conference, EPEC 2009, 2009, doi: 10.1109/EPEC.2009.5420809.

[30] A. Abdrabou, "A Wireless Communication Architecture for Smart Grid Distribution Networks," IEEE Syst. J., vol. PP, no. 99, pp. 111, 2014, doi: 10.1109/JSYST.2014.2304291.

[31] K. S. Ibwe, E. A. Kalinga, N. H. Mvungi, H. Tenhunen, and V. Taajamaa, "The Impact of Industry Participation on Challenge Based Learning *," Int. J. Eng. Educ., vol. 34, no. 1, pp. 187-200, 2018.

[32] E. A. Kalinga, K. S. Ibwe, and N. H. Mvungi, "Active Learning through Smart Grid Model Site in Challenge Based Learning Course," vol. 16, no. 3, pp. 53-64, 2018.

[33] Y. Andegelile, H. Maziku, N. Mvungi, and M. Kissaka, "Software Defined Communication Network Reliability for Secondary Distribution Power Grid," Int. J. Smart Grid - ijSmartGrid, vol. 4, no. 3, 2020.

[34] R. Fontes, M. N. Mahfoudi, W. Dabbous, and T. Turletti, "How Far Can We Go? Towards Realistic Software-De fi ned Wireless Networking Experiments," no. June, 2017, doi: 10.1093/comjnl/bxx023. 